\title{
Itô Atlas, its application to mathematical finance and to exponentiation of infinite dimensional Lie algebras
}

\author{
Paul Malliavin \\ 10 rue Saint Louis en l'Isle, 75004, Paris, sli@ccr.jussieu.fr
}

\section{Introduction}

The names "charts" or "atlas" have their origin in geography; it is of importance to represent "faithfully" on a sheet of paper a town, a province, on a region of the Earth; the atlas is a collection of charts such that every point of the Earth appears at least one time in the range of a chart of this collection.

In a more mathematical way the representation by longitude and latitude is described as follows : on the two dimensional sphere $S^{2}$, choose a North Pole $N$ and a half meridian of reference (Greenwich); denote $S_{0}^{2}$ the open subset of $S^{2}$ constituted by the complement of the reference half meridian; then the longitude, latitude $(\phi, \theta) \in] 0,2 \pi[\times] 0, \pi\left[\right.$ defines a local chart a bijective map $u_{0}$ of the domain of the chart, that is the open subset $] 0,2 \pi[\times] 0, \pi\left[\subset R^{2}\right.$ onto the range of the local chart, that is the open subset $S_{0}^{2} \subset S^{2}$; by choosing another North Pole and another reference half meridian we can construct another open subset $S_{1}^{2}$ of $S^{2}$ such that $S^{2}=S_{0}^{2} \cup S_{1}^{2}$; denote $u_{1}$ the corresponding local chart; then the two local charts $u_{0}, u_{1}$ constitute an atlas of $S^{2}$. This representation is faithful in the sense that

A function $f$ defined on $S^{2}$ is differentiable if and only if $f_{i}:=f \circ u_{i}, \quad i=$ 0,1 are differentiable; furthermore all differential geometric computations on $S^{2}$ can be effectively realized through the atlas.

Another possible atlas is the Mercator atlas: we denote $v_{N}$ the stereographic projection from the North pole which sends a sheet of paper into $S^{2}$ in one to one correspondence with the tangent plane to $S^{2}$ at the South pole; the range of $v_{N}$ is the open set of $S^{2}$ constituted by the complement of the North pole. Denote $v_{S}$ the stereographic projection from the South pole; then the Mercator atlas is the collection of the two charts $v_{N}, v_{S}$. For the geography it is possible to study the Earth equally well in each of these atlas; the choice between them is a question of convenience: the Mercator atlas preserves the angles and the longitude-latitude atlas refer to coordinates which can be immediately obtained from observations. 
Turn now to the same concepts for a $d$ dimensional manifold $\mathcal{M}$; a chart $\phi_{\alpha}$ will be a continuous injective map of an open subset of $O_{\alpha} \subset R^{d}$ such that its range $\phi_{\alpha}\left(O_{\alpha}\right)$ is an open set of $\mathcal{M}$; every point of $\mathcal{M}$ is in the range of a chart: $\cup_{\alpha} \phi_{\alpha}\left(O_{\alpha}\right)=\mathcal{M}$. Finally the differentiable structure on $\mathcal{M}$ will be defined as the "image" of the classical differential structure on $O_{\alpha}$; more precisely a function $f: \mathcal{M} \mapsto R$ is differentiable if and only if $f \circ \phi_{\alpha} \in C^{1}\left(O_{\alpha}\right) \forall \alpha$; the implementation of this definition can be done assuming the coherence hypothesis, that is: $\phi_{\beta}^{-1} \circ \phi_{\alpha} \in C^{1}\left(O_{\alpha}\right)$.

A classical theory of Banach modeled manifold exits where the $O_{\alpha}$ are now open subsets of a fixed Banach space $B$.

Lie algebra appearing in mathematical physics, as the Virasoro algebra $\mathcal{V}$, are infinite dimensional; the physical prerequisite of symplecticity determines on $\mathcal{V}$ a unique Hilbertian metric which has low regularity; this low regularity implies that the exponentiation from $\mathcal{V}$ to a propective "infinite dimensional group" cannot be realized into the context of Banach modeled manifold. A new concept of differential geometry has to be built in order to fit this infinite dimensional challenge: this will be sketched in the second part of this paper.

Let us justify now the first part. It can be thought that the "state space" of a financial market is an abstract manifold $\mathcal{M}$. This manifold $\mathcal{M}$ can be given through the choice of some model. We take a phenomenological point of view: a broker, from the simple observation of the market, wants to obtain, model free, some information on the structure of $\mathcal{M}$. For instance he will inquire: is it possible to compute econometrically the pathwise sensitivities? The computation of the Greeks require the differentiation of the coefficients the driving SDE. How could be possible to differentiate when we know this SDE only on the path on $\mathcal{M}$ describing the market evolution!

Itô calculus answers these questions coming from two different worlds.

\section{Itô's Atlas}

Given a "smooth manifold" $S$ (finite or infinite dimensional) and an "elliptic differential operator" on $S$, consider the sample path of the associated diffusion $s_{\omega}(\tau), \tau \in[0,1], \omega \in \Omega$ being the probability space.

An Itô local chartis the map $\tau \mapsto s_{\omega}(\tau), \tau \in[0,1]$ : the domain of charts are the 1-dimensional segment $[0,1]$, the range are sample paths of the diffusion.

The "dimensionality" of the range is 1 , even if $S$ is infinitely dimensional; but the cardinality of the atlas is large: there are as many local charts as there are sample paths of the diffusion.

Theorem 1 (Localization of derivative). Given a smooth function $f$ on $S$, its derivative can be computed in an Itô local chart from the knowledge of $f$ on the range of this local chart

Proof. Consider a local chart $u: O \mapsto S$, in the classical sense: $O$ is an open subset of $R^{N}$, the range of $u$ is an open subset of $S$; let $f^{u}:=f \circ u$. 
Given a semi-martingale $\varphi(\omega, \tau)$ defined on $\Omega$, denote $\langle\varphi\rangle_{\tau}$ the associated increasing process and define $\operatorname{Vol}_{\tau}(\varphi, \varphi)=\frac{d}{d \tau}\left(\langle\varphi\rangle_{\tau}\right)$; then $\operatorname{Vol}_{\tau}(\varphi, \varphi)$ is pathwise computable; define by polarization $\operatorname{Vol}(\phi, \psi)=\frac{1}{4}(\operatorname{Vol}(\phi+\psi, \phi+\psi)-$ $\operatorname{Vol}(\phi-\psi, \phi-\psi))$; then by Itô Calculus

$$
\frac{\partial f^{u}}{\partial \xi^{k}}=\frac{\operatorname{Vol}(\phi, \psi)}{\operatorname{Vol}(\phi, \phi)}, \phi=\left[u^{-1}\left(s_{\omega}\right)\right]^{k}, \psi=f\left(s_{\omega}\right)
$$

We split this paper in two parts which can be read independently one of the other.

\section{Computation of pathwise sensitivities in mathematical finance}

Let $p$ be the asset price, we will assume that $p(t)$ is a continuous semimartingale satisfying the SDE

$$
d p(t)=\sigma(t) d W(t)+b(t) d t
$$

where $W$ is a Brownian motion on a filtered probability space $\left(\Omega,\left(\mathcal{F}_{t}\right)_{t \in[0, T]}\right.$, $P), \sigma$ and $b$ are stochastic processes such that $E\left[\int_{0}^{T} \sigma^{4}(t) d t+\int_{0}^{T} b^{2}(t) d t\right]<$ $\infty$. The random function $\sigma^{2}(t)$ is the spot volatility. We want to propose a pathwise econometrical computation of the spot volatility $[4,13]$.

\subsection{Fourier econometrical computation of spot volatility}

By change of the origin of time and rescaling the unit of time we reduce ourselves to the case where the time window is $[0,2 \pi]$. Given a function $\phi$ on the circle $S^{1}$, we consider its Fourier transform, defined by

$$
\mathcal{F}(\phi)(k)=: \frac{1}{2 \pi} \int_{0}^{2 \pi} \phi(\vartheta) \exp (-i k \vartheta) d \vartheta, \quad \text { for } k \in \mathbf{Z} .
$$

Define

$$
\mathcal{F}(d \phi)(k)=: \frac{1}{2 \pi} \int_{] 0,2 \pi[} \exp (-i k \vartheta) d \phi(\vartheta),
$$

then by integration by part

$$
\mathcal{F}(\phi)(k)=\frac{i}{k} \times\left[\frac{1}{2 \pi} \phi(2 \pi)-\phi(0)-\mathcal{F}(d \phi)(k)\right] .
$$

Given two functions $\Phi, \Psi$ on the integers their Bohr convolution product is defined by

$$
\left(\Phi *_{B} \Psi\right)(k)=: \lim _{N \rightarrow \infty} \frac{1}{2 N+1} \sum_{s=-N}^{N} \Phi(s) \Psi(k-s) .
$$


Theorem 2. Consider a process p satisfying assumption (2); then we have

$$
\frac{1}{2 \pi} \mathcal{F}\left(\sigma^{2}\right)=\Phi *_{B} \Phi, \text { where } \Phi(k):=\mathcal{F}(d p)(k),
$$

the limit corresponding to the definition of $\Phi *_{B} \Phi$ is attained in probability.

\subsection{A reduced sensitivity: the feedback price-volatility rate}

We shall firstly discussed a reduced sensitivity which has the advantage to be computable through an ordinary ODE, when classical sensitivities require the integration of SDE. Assume that the price of the considered asset is given by a geometric martingale:

$$
d S_{W}(t)=\sigma\left(S_{W}(t)\right) d W(t)
$$

where $W$ is a Brownian motion, $\sigma$ is a fixed but unknown smooth function depending only upon the price.

The classical pathwise Greek Delta $\zeta_{W}(t)$ is defined as the solution of the linearized SDE

$$
d \zeta_{W}(t)=\left(\sigma^{\prime}\left(S_{W}(t)\right) d W((t)) \zeta_{W}(t)\right.
$$

We associate to $\zeta(t)$ the rescaled variation defined as

$$
z(t)=\frac{\zeta(t)}{\sigma\left(S_{W}(t)\right)}
$$

Theorem 3 (price-volatility feedback rate [5]). The rescaled variation is a derivable function of $t$; its logarithmic derivative $\lambda(t)$ will be called the price-volatility feedback rate function. Then

$$
\lambda(t)=-\frac{1}{2}\left(\sigma \sigma^{\prime \prime}\right)\left(S_{W}(t)\right) ; \quad z(t)=\exp \left(\int_{s}^{t} \lambda(\tau) d \tau\right) z(s) .
$$

Remark: $\lambda(t)<0$ corresponds to a liquid market.

We suppose that we do not know the explicit expression of the function $\sigma$; we want to obtain from the pathwise observation of the market evolution an econometrical computation in real time of $\lambda(t)$. Making the change of variables

$$
x_{W}(t)=\log \left(S_{W}(t)\right) ; \quad a(x)=\exp (-x) \sigma((\exp (x)),
$$

then $x_{W}(t)$ satisfies the following SDE:

$$
d x_{W}(t)=a\left(x_{W}(t)\right) d W(t)-\frac{1}{2} a^{2}\left(x_{W}(t)\right) d t
$$

The price-volatility feedback rate has in logarithmic coordinate the following expression :

$$
\lambda=-\frac{1}{2}\left(a^{\prime} a+a a^{\prime \prime}\right)
$$

Denote $\star$ the Itô contraction, which can be compute pathwise by (3). 
Theorem 4 (Econometrical computation of the feed-back rate). Define $d x \star d x:=A(t), d A \star d x:=B(t), d B \star d x:=C(t)$, then

$$
\lambda(t)=\frac{3}{8} \frac{B^{2}}{A^{3}}-\frac{1}{4} \frac{B}{A}-\frac{1}{2} \frac{C}{A^{2}}
$$

Remark. The computations are made in the following order; firstly compute the Fourier coefficients of $x$; by applying (3) we obtain the Fourier coefficients of $A$; denote $\alpha$ a parameter, then the Fourier coefficients of $x+\alpha A$ are known; by applying again (3) we get the Fourier coefficients of $\operatorname{Vol}(x+\alpha A)$; as $4 B=\operatorname{Vol}(x+A)-\operatorname{Vol}(x-A)$ we get the Fourier coefficients of $B$; then the Fourier coefficients of $x+\alpha B$ are known and by using again (3) we get the Fourier coefficients of $\operatorname{Vol}(x+\alpha B)$; then the identity $4 C=\operatorname{Vol}(x+B)-\operatorname{Vol}(x-B)$ gives the Fourier coefficients of $C$.

\subsection{Outline of computations of general pathwise sensitivities}

We sketch some facts which are developed in [14]. Start firstly from the observation that for the infinitesimal generator $\mathcal{L}$ of the risk free measure the drift can be computed in term of the volatility: in the case of Black-Scholes model $\mathcal{L}$ has a vanishing drift; in the case of HJM model of the interest curve the drift is explicitly expressed in term of the volatility matrix. As the volatility matrix can be econometrically pathwise computed, it results that $\mathcal{L}$ can be pathwised computed.

Using (1) the derivatives of the coefficients of $\mathcal{L}$ can be computed; then the pathwise Greek Delta can be computed, at the price of solving numerically an SDE.

Compared to the computation of the feedback price-volatility rate, these operations have the advantage to involve one step of computation of volatility less but from the other hand there are leading to an SDE which is numerically more instable than the ODE driving the reduced variation.

\section{Differential geometry on Hölderian Jordan curves}

When in 1870-1890 Sophus Lie made his foundational work on what is known now as the theory of finite dimensional Lie algebras, he splitted his progression along three main theorems; he called the Third Theorem the fact that given a Lie algebra $\mathcal{G}$, then it is possible to construct a group $G$ having for Lie algebra $\mathcal{G}$. Let us call this statement the exponentiation problem for the Lie algebra $\mathcal{G}$. It took about twenty years to Sophus Lie to solve in full generality the exponentiation problem for finite dimensional Lie algebras.

For infinite dimensional Lie algebras $\mathcal{G}$ coming from mathematical physics, infinitesimal representations of $\mathcal{G}$ are known from around twenty years back; the exponentiation problem can be thought as follows: given an infinitesimal 
representation $\lambda$ of $\mathcal{G}$, find a probability measure $\mu_{\lambda}$, such that $\lambda$ integrates into a representation on $L_{\mu_{\lambda}}^{2}$; this situation is furthermore complicated by a the necessity to prove a Cameron-Martin theorem.

It is clear that in this situation the Banach-model manifold theory is hopeless. We shall emphasize the advantage of the Itô atlas in a CFT (conformal field theory) context.

\subsection{Jordan curves and their parameterizations}

A Jordan curve $\Gamma$ is the range of a continuous injective map $\phi$ of the circle $S^{1}$ into the plane. Let $h \in H\left(S^{1}\right)$ be the group of homeomorphisms of $S^{1}$; then $\phi$ and $\phi \circ h$ define the same Jordan curve.

\subsection{Holomorphic parameterization}

The Jordan curve $\Gamma$ splits the complex plane in two simply connected domains $\Gamma^{+}, \Gamma^{-}$; by the Riemann mapping theorem there exists an holomorphic map $f^{+}$realizing a bijective map of the unit disk $D$ onto $\Gamma^{+}$; by Caratheodory $f^{+}$ has a continuous extension $\bar{f}^{+}$to $\bar{D}$; the restriction $\bar{f}^{+}$to $\partial D$, the circle $S^{1}$, defines the holomorphic parameterization which is unique up to $h \in \mathcal{H}\left(S^{1}\right)$ the Poincaré group of holomorphic automorphisms of $D$.

\subsection{Conformal welding parameterization}

The composition $g: \theta \mapsto\left(\left[\bar{f}^{+}\right]^{-1} \circ \bar{f}^{-}\right)\left(e^{i \theta}\right)$ defines $g \in H\left(S^{1}\right)$, where $H\left(S^{1}\right)$ denotes the group of homeomorphisms of the circle. Denote $G$ the group of $C^{\infty}$ diffeomorphisms of $S^{1}$, then by Beurling-Ahlfors [6] the set of $C^{\infty}$ Jordan curves is isomorphic to $\mathcal{H} \backslash G / \mathcal{H}$.

\subsection{Canonical Hilbert norm on $\mathcal{G}$}

The Lie algebra $\mathcal{G}$ of $G$ the group of smooth diffeomorphisms of the circle is constituted of smooth vector fields on $S^{1}$; granted the parallelism $\frac{d}{d \theta}$ we have a linear isomorphism $\mathcal{G} \simeq C^{\infty}\left(S^{1}\right)$, the vector space of smooth functions on $S^{1}$.

$\mathcal{H}$ denotes the restriction to the circle of the Poincaré group of homographic transformations

$$
z \mapsto \frac{a z+b}{\bar{b} z+\bar{a}}, \quad|a|^{2}-|b|^{2}=1 ;
$$

the Lie algebra of $\mathcal{H}$ is $\mathrm{su}(1,1)$ which is generated by the three vector fields $\cos \theta, \sin \theta, 1$.

There exists [2] a unique semi-Hilbertian metric on $\mathcal{G}$ invariant by the adjoint action of $s u(1,1)$. Therefore the set of $C^{\infty}$ Jordan curves $\simeq \mathcal{H} \backslash G / \mathcal{H}$ has a unique Hilbert structure. 
The associated canonical Brownian motion on $\mathcal{G}$ has the following expression:

$$
x(t)=\sum_{k>1} \frac{1}{\sqrt{k^{3}-k}}\left(x_{2 k}(t) \cos k \theta+x_{2 k+1}(t) \sin k \theta\right),
$$

where $x_{*}(t)$ is an infinite sequence of scalar valued independent Brownian motions.

\subsection{Exponentiating Brownian motion on $\mathcal{G}$}

Fix $\rho \in] 0,1[$ and use the Abel regularization:

$$
{ }^{\rho} x_{t}(\theta):=\sum_{k>1} \frac{\rho^{k}}{\sqrt{k^{3}-k}}\left(x_{2 k}(t) \cos k \theta+x_{2 k+1}(t) \sin k \theta\right),
$$

then ${ }^{\rho} x_{t}(*) \in C^{\infty}\left(S^{1}\right)$; by the theory of Stochastic flow of diffeomorphisms there exits a solution of the SDE

$$
d_{t}\left({ }^{\rho} g_{x_{t}}(\theta)\right)=\left(d_{t}{ }^{\rho} x_{t}\right)\left({ }^{\rho} g_{x_{t}}(\theta)\right), \quad{ }^{\rho} g_{x_{0}}(\theta)=\theta
$$

which is a $C^{\infty}$ diffeomorphism of $S^{1}$. Furthermore by Beurling-Ahlfors is associated to the process $t \mapsto^{\rho} g_{x_{t}}$ a non markovian process $t \mapsto^{\rho} \Gamma_{x_{t}}$ on the space of $C^{\infty}$ Jordan curves.

Theorem $5([\mathbf{3}, \mathbf{1 1}])$. The limit when $\rho \rightarrow 1$ of ${ }^{\rho} g_{x_{t}}$ exists $=: g_{x_{t}}$; and $g_{x_{t}}$ is an homeomorphism which is Hölderian of exponent $\exp (-2 t)$.

Theorem 6 ([2]). The process $t \mapsto{ }^{\rho} \Gamma_{x_{t}}$ converges to a diffusion $t \mapsto \Gamma_{x_{t}}$ on the space of Hölderian Jordan curves, starting at time $t=0$ from the unit circle.

\section{6 $C^{\infty}$ differential geometry "on" Hölderian Jordan curves}

Denote $\mu_{t}$ the law of $\Gamma_{x_{t}}$; to study the Cameron-Martin Theorem for $\mu_{t}$ it is known $[9,10]$ that infinite dimensional Riemannian geometry has to be introduced.

It would be a non sense to try to enforce a Banach-model differentiable structure on the space of Jordan curves.

We could proceed by using the notion of dressed up trajectory $\tilde{\Gamma}_{x_{*}}$ of $\Gamma_{x, *}$ which is the lift to the $C^{\infty}$ jet bundle of $\Gamma_{x, *}$ provided by iterative applications of the formula (1). As effective computations on jet bundles are difficult, even in finite dimension, we shall not follow this approach.

We shall use the lift to the orthonormal frame bundle; this lift will provide the same information that the lift to the $C^{\infty}$ jet bundle and will enjoy computational flexibility. 


\subsection{Orthonormal frame bundle of over $G_{\rho}$}

Denote $\mathcal{G}_{\rho}$ the Hilbert space associated to $(* \mid *)_{\rho}$ the scalar product associated to the regularized metric which has been made positive definite by introducing on the 3 -dimensional subspace $V$ generated by $1, \sin \theta, \cos \theta$ the hilbertian metric generated by the $L^{2}\left(S^{1}\right)$ norm, $V$ being furthermore orthogonal to all others trigonometrical functionals.

Denote $\mathcal{U}_{\rho}$ the group of unitary operators of $\mathcal{G}_{\rho}$. Denote $O\left(G_{\rho}\right)$ the bundle of orthonormal frame of $G_{\rho}$ : a frame is an hilbertian isomorphism of the tangent plane $T_{g}\left(G_{\rho}\right)$ into the Hilbert space $\mathcal{G}_{\rho}$.

We have a canonical section of $O\left(G_{\rho}\right)$ given by

$$
g \mapsto r_{g}^{0}, \quad r_{g}^{0}(\zeta) \text { where } \zeta=\exp \left(\epsilon r_{g}^{0}(\zeta)\right) g \quad, \quad \epsilon \rightarrow 0 ;
$$

in another words $r_{g}^{0}$ is the inverse map of the infinitesimal exponential map.

The natural action of $\mathcal{U}_{\rho}$ acts on $\mathcal{G}_{\rho}$ prolongate at the level of frame as $U \times r^{0}=U \circ r^{0}$. In this way we have the following canonical isomorphism

$$
O\left(G_{\rho}\right) \simeq \mathcal{U}_{\rho} \times G_{\rho}
$$

\subsection{Christofell symbols}

Two parallel transports in the direction $z$ coexist: $\mathcal{T}_{z}^{1}:=$ the algebraic transport obtained by composition of homeomorphisms, $\mathcal{T}_{z}^{2}:=$ the Riemannian Levi-Civita parallel transport; then $\mathcal{T}_{z}^{2}-\mathcal{T}_{z}^{1}$ defines $\Gamma(z) \in$ Endo$\left.\operatorname{morphism}\left(\mathcal{G}_{\rho}\right)\right)$; this key endomorphism is determined by the next Theorem.

Theorem 7 (see [8]). The Riemannian Levi-Civita connection on $G_{\rho}$ is expressed in the canonical moving frame $r_{*}^{0}$ by the following $(1,2)$ constant tensor

$$
{ }^{\rho} \Gamma_{i j}^{k}=\frac{1}{2}\left(\left(\left[{ }^{\rho} e_{i},{ }^{\rho} e_{j}\right] \mid{ }^{\rho} e_{k}\right)_{\rho}-\left(\left[{ }^{\rho} e_{j},{ }^{\rho} e_{k}\right] \mid{ }^{\rho} e_{i}\right)_{\rho}+\left(\left[{ }^{\rho} e_{k},{ }^{\rho} e_{i}\right] \mid{ }^{\rho} e_{j}\right)_{\rho}\right) ;
$$

denote ${ }^{\rho} \boldsymbol{\Gamma}_{i}$ the endomorphism of $\mathcal{G}$ corresponding to the last two indices, then ${ }^{\rho} \boldsymbol{\Gamma}_{i} \in \operatorname{so}\left(\mathcal{G}_{\rho}\right)$.

Proof. As the antisymmetry property implies that connection preserves the Riemannian metric we have only to prove that it has no torsion which means that

$$
{ }^{\rho} \Gamma_{i j}^{k}-{ }^{\rho} \Gamma_{j i}^{k}=\left(\left[{ }^{\rho} e_{i},{ }^{\rho} e_{j}\right] \mid{ }^{\rho} e_{k}\right)_{\rho},
$$

identity which results by direct inspection from (11).

Corollary 8. The process $t \mapsto{ }^{\rho} g_{x}(t)$ is the Brownian motion on $G_{\rho}$

As we have 


$$
\begin{aligned}
2[\cos k \theta, \cos p \theta] & =(k-p) \sin (k+p) \theta+(k+p) \sin (k-p) \theta \\
2[\sin k \theta, \sin p \theta] & =(p-k) \sin (p+k) \theta+(p+k) \sin (k-p) \theta \\
2[\cos k \theta, \sin p \theta] & =(p-k) \cos (p+k) \theta+(p+k) \cos (p-k) \theta
\end{aligned}
$$

we deduce the following expression for the Christofell symbol in the complex exponential basis;

For $p, k \geq 2$,

$$
\begin{aligned}
& { }^{\rho} \Gamma\left(e^{i p \theta}\right) e^{i k \theta}=i \frac{(2 p+k) \alpha(k)}{\alpha(p+k)} e^{i(p+k) \theta} \\
& { }^{\rho} \Gamma\left(e^{i p \theta}\right) e^{-i k \theta}=-i \rho^{2 p}(p+k) 1_{k \geq p+2} \times e^{-i(k-p) \theta}
\end{aligned}
$$

where $\alpha(k)=k^{3}-k$.

\subsection{Riemannian parallelism on the frame bundle $O\left(G_{\rho}\right)$}

We have an algebraic parallelism on $\mathcal{U}_{\rho} \times G$ induced by the right invariant Lie algebra of the product group $\operatorname{so}\left(\mathcal{G}_{\rho}\right) \times \mathcal{G}$; a constant vector field in the algebraic parallelism is of the form

$$
\begin{gathered}
\left.(\zeta)_{u, g}=(\exp \epsilon \ddot{\zeta}) \times U, \exp (\epsilon \dot{\zeta}) \times g\right), \epsilon \rightarrow 0, \\
\ddot{\zeta} \in \operatorname{so}\left(\mathcal{G}_{\rho}\right), \quad \dot{\zeta} \in \mathcal{G}_{\rho}
\end{gathered}
$$

The 1-differential forms describing the passage from the algebraic to the Riemannian parallelism are:

$$
\begin{gathered}
<\zeta, \dot{\sigma}>_{U, g}=U(\dot{\zeta}), \\
<\zeta, \ddot{\sigma}>_{U, g}=U \circ\left(\ddot{\zeta}+{ }^{\rho} \boldsymbol{\Gamma}\left(U^{*}(\dot{\zeta})\right)\right) \circ U^{*}
\end{gathered}
$$

where $U^{*}$ is the adjoint (and the inverse) of $U$.

The parallelism differential forms do not depend upon $g$, fact which corresponds to the invariance of the Riemannian metric under the right $G$ action.

\subsection{Structural equations [8]}

The coboundary of the parallelism differential forms are

$$
\begin{aligned}
& <\zeta_{1} \wedge \zeta_{2}, d \dot{\sigma}>=\ddot{\sigma}\left(\zeta_{1}\right)\left(\dot{\sigma}\left(\zeta_{2}\right)\right)-\ddot{\sigma}\left(\zeta_{2}\right)\left(\dot{\sigma}\left(\zeta_{1}\right)\right) \\
& <\zeta_{1} \wedge \zeta_{2}, d \ddot{\sigma}>=\ddot{\sigma}\left(\zeta_{1}\right) \ddot{\sigma}\left(\zeta_{2}\right)-\ddot{\sigma}\left(\zeta_{2}\right) \ddot{\sigma}\left(\zeta_{1}\right)+{ }^{\rho} \mathbf{R}(\dot{\sigma}(\zeta) \wedge \dot{\sigma}(\zeta))
\end{aligned}
$$

where ${ }^{\rho} \mathbf{R}$ is the Riemannian curvature propagated by tensorial variance from its definition in $U=$ Identity given as

$$
{ }^{\rho} \mathbf{R}\left(\dot{\zeta}_{1} \wedge \dot{\zeta}_{2}\right)={ }^{\rho} \boldsymbol{\Gamma}\left(\dot{\zeta}_{1}\right){ }^{\rho} \boldsymbol{\Gamma}\left(\dot{\zeta}_{2}\right)-{ }^{\rho} \boldsymbol{\Gamma}\left(\dot{\zeta}_{2}\right)^{\rho} \boldsymbol{\Gamma}\left(\dot{\zeta}_{1}\right)-{ }^{\rho} \boldsymbol{\Gamma}\left(\left[\dot{\zeta}_{1}, \dot{\zeta}_{2}\right]\right) .
$$




\subsection{Change of parameterization through horizontal parameterization}

The canonical horizontal lifting of ${ }^{\rho} g_{x}(t)$ to $O\left(G_{\rho}\right)$ is the process $\left(\Omega_{t}, g_{x}(t)\right)$ where $\Omega_{*}$ is obtained by solving the stochastic differential system

$$
\begin{aligned}
d z(t) & =\Omega_{t}(d x(t)) \\
d \Omega_{t} & =\left(-\sum_{k \geq-1}{ }^{\rho} \boldsymbol{\Gamma}\left({ }^{\rho} e_{k}\right) d z_{k}(t)+\frac{1}{2} \sum_{k \geq-1}\left({ }^{\rho} \boldsymbol{\Gamma}\left({ }^{\rho} e_{k}\right)\right)^{2} d t\right) \Omega_{t}
\end{aligned}
$$

with $\Omega_{0}=\mathrm{Id}$

We could look to the system (15) as $x$ given and $z$ must be determined by an implicit SDE, which is difficult to solve.

As the mapping $x \mapsto z$ is an isomorphism of probability space, it is better to take as initial data $z$ then solve the second SDE (15), then obtain $x$ by $d x(t)=\Omega_{t}^{-1}(d z(t))$ and finally $g_{x}$ by solving $d g_{x}(t)=* d x(t) \circ g_{x}(t)$.

\subsection{Stochastic Calculus of Variations on the horizontal flow}

Let $a \mapsto{ }_{z} \Theta_{t \leftarrow 0}(a)$ the stochastic flow on $O\left(G_{\rho}\right)$ defined by solving the Stratanovitch SDE

$$
<* d r_{z}, \dot{\sigma}>=d z(t), \quad<* d r_{z}, \ddot{\sigma}>=0, \quad r_{z}(0)=a
$$

Given $a(\epsilon)$ a differentiable curve on $O\left(G_{\rho}\right)$, the Jacobian flow ${ }_{z} \Theta^{\prime}{ }_{t \leftarrow 0}$ is defined by

$$
\begin{aligned}
\frac{d}{d \epsilon_{\epsilon=0}}{ }_{z} \Theta_{t \leftarrow 0}(a(\epsilon)) & =:{ }_{z} \Theta^{\prime}{ }_{t \leftarrow 0}\left(a^{\prime}(0)\right) \\
<{ }_{z} \Theta^{\prime}{ }_{t \leftarrow 0}\left(a^{\prime}(0)\right), \dot{\sigma}> & =: \dot{h}(t), \\
<{ }_{z} \Theta^{\prime}{ }_{t \leftarrow 0}\left(a^{\prime}(0)\right), \ddot{\sigma}> & =: \ddot{h}(t) .
\end{aligned}
$$

Theorem 9.

$$
\begin{aligned}
& d_{t} \dot{h}+\frac{1}{2}{ }^{\rho} \operatorname{Ricci}_{\Omega_{z}(t)}(\dot{h}(t)) d t=\ddot{h}(t) d z_{t}, \\
& d_{t} \ddot{h}={ }^{\rho} \mathbf{R}_{\Omega_{z}(t)}\left(d z_{t} \wedge \dot{h}(t)\right)+{ }^{\rho} \tilde{f}_{\Omega_{\Omega_{z}(t)}}(\ddot{h}(t)) d t,
\end{aligned}
$$

where $\tilde{\mathbf{R}}$ is the curvature tensor considered as defining an endomorphism of $\operatorname{so}\left(\mathcal{G}_{\rho}\right)$, the space of antihermitian bounded operators over $\mathcal{G}_{\rho}$.

\subsection{Integration by part for the regularized metric}

Theorem 10 (Theorem of integration by part $([\mathbf{7}, \mathbf{9}, 11]))$. Let $v \in \mathcal{G}_{\rho}$; assume that there exists $\delta>0$ such that, in the sense of the order of hermitian operator on $\mathcal{G}_{\rho}$, the following inequality holds true ${ }^{\rho}$ Ricci $\geq-\delta \times$ Identity, then 


$$
\begin{aligned}
\frac{d}{d \epsilon_{\epsilon=0}}\left(E\left(f\left({ }^{\rho} g_{x}\left(t_{0}\right) \exp \left(\epsilon v_{0}\right)\right)\right)\right) & =E\left(f\left({ }^{\rho} g_{x}\left(t_{0}\right)\right) k(x)\right), \text { where } \\
E\left(|k(x)|^{2}\right) & \leq \frac{\exp \left(\delta t_{0}\right)-1}{\delta t_{0}^{2}}\left\|v_{0}\right\|_{\rho}^{2} .
\end{aligned}
$$

Proof. We follow [12]; an infinitesimal euclidean motion of the $\mathcal{G}_{\rho}$-cylindrical Brownian motion is defined

$$
z \mapsto y, y(t)=\int_{0}^{t} \exp \left(\epsilon q_{s}\right) d z(s)+\epsilon \int_{0}^{t} w(s) d s
$$

where $s \mapsto q_{s}$ is an adapted functional with values in $\operatorname{so}\left(\mathcal{G}_{\rho}\right)$ and where the map $* \mapsto w_{*}$ is an adapted functional with values in $L^{2}\left(\left[0, t_{0}\right] ; \mathcal{G}_{\rho}\right)$; define the derivative

$$
D_{q, w}\left({ }_{*} \Theta_{t_{0} \leftarrow 0}\right):=\zeta\left(t_{0}\right), \quad \zeta(t):=\int_{0}^{t}{ }_{*} \Theta_{t_{0} \leftarrow s}^{\prime}\left(q_{s}, w_{s}\right) d s .
$$

We choose $(q, w)$ by solving the system

$$
d_{t} v+\frac{1}{2} \operatorname{Ricci}(v)=w, v(0)=v_{0}, \quad d q=\mathbf{R}(* d z \wedge v), q(0)=0 ;
$$

then taking $\zeta$ from $(a)$ and $(b)$ we get,

$$
\begin{aligned}
& d_{t} \dot{\zeta}+{ }^{1}{ }^{\rho} \operatorname{Ricci}_{\Omega_{z}(t)}(\dot{\zeta}(t)) d t=(q-\ddot{\zeta}(t)) d z_{t}+w d t, \\
& d_{t} \ddot{\zeta}={ }^{\rho} \mathbf{R}_{\Omega_{z}(t)}\left(* d z_{t} \wedge \dot{\zeta}(t)\right)
\end{aligned}
$$

which leads to the following remarkable expression

$$
\dot{\zeta}=v, \ddot{\zeta}=q .
$$

Denote $A_{t \leftarrow 0}$ the resolvent of the first equation (b), take

$$
\begin{gathered}
w(t)=-\frac{1}{t_{0}} A_{t \leftarrow 0}\left(v_{0}\right) \\
w\left(t_{0}\right)=A_{t_{0} \leftarrow 0}\left(v_{0}\right)+\int_{0}^{t_{0}} A_{t_{0} \leftarrow t} h(t) d t=0
\end{gathered}
$$

this last equality meaning that the "jump" resulting from the derivative at $t=0$ have been completely sweep out at time $t_{0}$. Let us emphasize that the equation $(d)$ gives rise to an adapted $h$. As the infinitesimal rotations preserves the gaussian measure we have $D_{q, h}$ as the same formula of integration by part as $D_{0, h}$ formula which is provided by Girsanov:

$$
k(z)=\frac{1}{t_{0}} \int_{0}^{t_{0}}\left(A_{t \leftarrow 0}\left(v_{0}\right) \mid d z_{t}\right) ;
$$


by Itô energy identity of stochastic integral we have

$$
E\left(|k(z)|^{2}\right) \leq\left\|v_{0}\right\|_{\mathcal{G}_{\rho}}^{2} \frac{1}{t_{0}^{2}} \int_{0}^{t_{0}} \exp (\delta t) d t
$$

expression equal to the r.h.s. of (17).

\subsection{Cameron-Martin for the Brownian on $G$}

Theorem 11. Assume that there exists $\delta$ such that for all $\rho<1$,

$$
\left({ }^{\rho} \operatorname{Ricci} \zeta \mid \zeta\right)_{\rho} \geq-\delta\|\zeta\|_{\rho}^{2} \quad \forall \zeta
$$

and that for all $\rho<1$ the following SDE is resoluble and that its solution is a unitary operator

$$
d^{\rho} \Omega_{t}=\left(-\sum_{k \geq-1}{ }^{\rho} \boldsymbol{\Gamma}\left({ }^{\rho} e_{k}\right) d z_{k}(t)+\frac{1}{2} \sum_{k \geq-1}\left({ }^{\rho} \boldsymbol{\Gamma}\left({ }^{\rho} e_{k}\right)\right)^{2} d t\right){ }^{\rho} \Omega_{t} .
$$

Then under these hypothesis (20) holds true:

$$
\begin{aligned}
\left.\frac{d}{d \epsilon_{\epsilon=0}} E\left(f\left(g_{x}(t)\right) \exp \left(\epsilon v_{0}\right)\right)\right) & =E\left(f\left(g_{x}(t)\right) k(x)\right), \\
E\left(|k(x)|^{2}\right) & \leq \frac{\exp \left(\delta t_{0}\right)-1}{\delta t_{0}^{2}}\left\|v_{0}\right\|^{2} .
\end{aligned}
$$

Remark. The unique hypothesis where uniformity relatively to $\rho$ appears is (18).

\subsection{Existence of the horizontal stochastic transport}

We shall prove, for $\rho<1$ the existence of a solution of (19); remark that the SDE (19) stays invariant in law by a rotation of the circle.

\section{Lemma 12.}

$$
\mathcal{C}:=\sum_{p \geq 2}\left({ }^{r} \boldsymbol{\Gamma}\left(\frac{\cos p \theta}{\sqrt{\alpha(p)}}\right)\right)^{2}+\left({ }^{r} \boldsymbol{\Gamma}\left(\frac{\sin p \theta}{\sqrt{\alpha(p)}}\right)\right)^{2}
$$

is a diagonal bounded operator and the coefficients on the diagonal are given by

$$
\lambda_{k}=\frac{1}{2} \sum_{p \geq 2}\left[-(p+k)^{2} \frac{\alpha(k-p)}{\alpha(p) \alpha(k)} 1_{k \geq p+2}-\frac{(2 p+k)^{2} \alpha(k)}{\alpha(p) \alpha(p+k)}\right] \rho^{p}
$$

Proof. The invariance in law of (19) under the action of $S^{1}$ implies that $\mathcal{C}$ commutes with the action of $S^{1}$ which is equivalent to say that $\mathcal{C}$ diagonalizes in the trigonometric basis. The expression of this diagonal matrix is obtained by direct computation. 
Lemma 13. The solution of (19) is given by the Picard series:

$$
\begin{gathered}
\Omega_{t}=\sum_{n \geq 0} \Delta_{n}(t), \\
\Delta_{0}(t)=\text { Identity, } \Delta_{n}(t)=\int_{0}^{t} \rho \boldsymbol{\Gamma}(d x(s)) \Delta_{n-1}(s)
\end{gathered}
$$

Proof. Define $M_{n}(t):=E\left(\Delta_{n}(t)\left(\Delta_{n}(t)\right)^{*}\right)$, then as before $M_{n}(t)$ is a diagonal matrix; by orthogonality of iterated Itô integrals we have : $M_{n}(t)=$ $\sum_{k} \frac{\rho^{2 k}}{\alpha(k)} A_{n, k}(t)$ where $A_{n, k}$ are the diagonal matrices defined by

$$
\begin{aligned}
A_{n, k}(t):={ }^{\rho} \boldsymbol{\Gamma}(\cos k \theta) \int_{0}^{t} M_{n-1}(s) & d s{ }^{\rho} \boldsymbol{\Gamma}(\cos k \theta) \\
& +{ }^{\rho} \boldsymbol{\Gamma}(\sin k \theta) \int_{0}^{t} M_{n-1}(s) d s{ }^{\rho} \boldsymbol{\Gamma}(\sin k \theta)
\end{aligned}
$$

\subsection{Curvatures of $S^{1}$-homeomorphism group (see [1])}

For $u, v \in \operatorname{diff}\left(S^{1}\right)$, the Riemann curvature has been defined as

$$
R(u, v):=\Gamma(u) \Gamma(v)-\Gamma(v) \Gamma(u)-\Gamma([u, v])
$$

Theorem 14. $2 R(\cos m \theta, \sin m \theta) \sin p \theta=-\lambda_{m, p} \cos p \theta$,

$$
\begin{gathered}
\lambda_{m, p}=1_{p \geq m+2} \frac{(m+p)^{2} \alpha(p-m)}{\alpha(p)}-\frac{(2 m+p)^{2} \alpha(p)}{\alpha(p+m)}+2 m p \\
\sum_{p \geq 2} \lambda_{m p}=-\frac{13}{6}\left(m^{3}-m\right)
\end{gathered}
$$

Theorem 15. The Ricci tensor is equal to $-\frac{13}{6} \times$ Identity

See for related results Bowick-Rajeev.

\section{References}

1. H. Airault. Riemannian connections and curvatures on the universal Teichmuller space. Comptes Rendus Mathématique, 341:253-258, 2005.

2. H. Airault, P. Malliavin, and A. Thalmaier. Canonical Brownian motion on the space of univalent functions and resolution of Beltrami equations by a continuity method along stochastic flows. J. Math. Pures et App., 83:955-1018, 2004.

3. H. Airault and J. Ren. Modulus of continuity of the canonic brownian motion "on" the group of diffemorphisms of the circle. J. Funct. Analysis, 196:395-406, 2002. 
4. E. Barucci, P. Malliavin, and M. E. Mancino. Harmonic Analysis methods for non parametric estimation of volatility: theory and applications. In Proc. Ritskumeisan Conference on Mathematical Finance, 2005. to appear in 2006.

5. E. Barucci, P. Malliavin, M. E. Mancino, and A. Thalmaier. The price-volatility feed-back rate, an implementable indicator of market stability. Math. Finance, 13:17-35, 2003.

6. A. Beurling and L. Ahlfors. The boundary correspondence under quasiconformal mappings. Acta Mathematica, 96:125-142, 1956.

7. J. M. Bismut. Large deviations and the Malliavin Calculus. Progress in Mathematics, 45, 1984. Birkhäuser, Boston.

8. A. B. Cruzeiro and P. Malliavin. Renormalized differential geometry on the path space: Structural equations, Curvatures. J. Funct. Analysis, 139:119-181, 1996.

9. B. Driver. Integration by part and quasi-invariance for heat measure over loop groups. J. Funct. Analysis, 149:470-547, 1997.

10. S. Fang. Integration by part for heat measures over loop groups. J. Math. Pures App., pages 877-894, 1999.

11. S. Fang. Canonical Brownian motion on the diffeomorphism group of the circle. J. Funct. Analysis, 196:162-179, 2002.

12. S. Fang and P. Malliavin. Stochastic Analysis on the path space of a riemannian manifold. J. Funct. Analysis, 118:249-274, 1993.

13. P. Malliavin and M. E. Mancino. Fourier series method for mesurement of multivariate volatilities. Finance Stoch., 6:49-61, 2002.

14. P. Malliavin and A. Thalmaier. Stochastic Calculus of Variations in Mathematical Finance. Springer, 2005. 142 pages. 